\title{
Colaborar con socios internacionales para innovar: implicaciones para empresas de servicios profesionales y tecnológicos
}

\author{
María Jesús Nieto ${ }^{1}$, Alicia Rodríguez y Luis Santamaría \\ Universidad Carlos III de Madrid, España
}

doi: $10.20420 /$ eni.2021.423

\begin{abstract}
Resumen
Las empresas de servicios intensivos en conocimiento juegan un papel fundamental como intermediarios y generadores de conocimiento en la economía actual. La comprensión, por tanto, de cómo estas empresas desarrollan innovaciones es de suma importancia. Entre las estrategias que contribuyen al desarrollo de innovaciones en estas empresas analizamos las colaboraciones tecnológicas. En este artículo resumimos las implicaciones de las colaboraciones internacionales para las empresas de servicios intensivos en conocimiento, diferenciando entre las de carácter profesional y las tecnológicas. Se tiene en cuenta la diversidad y la proximidad de los socios en la colaboración.
\end{abstract}

Palabras clave: colaboración internacional, diversidad de socios, proximidad de los socios, innovación.

Clasificación JEL: L14, O32, O33.

Agradecimiento: Los autores agradecen el apoyo financiero proporcionado por el Ministerio de Ciencia e Innovación [proyecto PID2019-106874GB-I00/AEI/10.13039/501100011033] y del Ministerio de Economía y Transformación Digital [proyecto ECO2017-87514-P].

Fuente de referencia: Rodríguez, A., Nieto, M. J., \& Santamaría, L. (2018). International collaboration and innovation in professional and technological knowledge-intensive services. Industry and Innovation, 25(4), 408-431.

\section{Introducción}

La colaboración internacional entre empresas es un fenómeno creciente, que ha suscitado un gran interés entre los académicos y que tiene importantes implicaciones para la gestión empresarial. En este contexto, la elección del socio es una decisión compleja y de vital importancia en tanto que tiene consecuencias relevantes para el resultado de la colaboración. Si, además, la colaboración se lleva a cabo entre empresas de distintos países surgen condicionantes tan decisivos como la procedencia geográfica o la posibilidad de colaborar de forma virtual. En este estudio recogemos las principales conclusiones del trabajo de Rodríguez, Nieto y Santamaría (2018), en el cual se analiza la colaboración tecnológica y la innovación en empresas de servicios intensivos en conocimiento (en adelante, KIBS).

Las KIBS representan en media el $18,8 \%$ del volumen de negocio en las economías europeas (excluyendo servicios financieros), siendo la tercera actividad en generación de valor añadido y la quinta en empleo (Eurostat, 2017). Por tanto, se trata de sectores estratégicos y muy relevantes en las economías actuales. En las KIBS gran parte del trabajo es de naturaleza intelectual, por lo que su principal activo es el capital humano altamente cualificado (Alvesson, 2004). Para este tipo de empresas, las redes juegan un papel fundamental para crear e intercambiar conocimiento. Por eso, la tendencia a colaborar e interactuar con otros agentes es muy útil para poder desarrollar innovaciones.

${ }^{1}$ Autor de correspondencia: mnieto@emp.uc3m.es. 
No obstante, los trabajos que han analizado este tipo de empresas subrayan que tanto la innovación como la propensión a colaborar difiere mucho entre los distintos tipos de KIBS (Pina \& Tether, 2016). En este ámbito es habitual distinguir entre empresas de servicios profesionales intensivos en conocimiento (en adelante P-KIBS) y empresas de servicios intensivos en conocimiento de carácter técnico (en adelante T-KIBS). Las P-KIBS incluyen empresas de servicios relacionados con consultoría de empresas, actividades legales, trabajos de arquitectura, ingeniería o investigación de mercados. En el caso de las TKIBS encontramos empresas relacionadas con la investigación y desarrollo experimental en ciencias e ingeniería, mantenimiento $\mathrm{y}$ reparación de maquinaria, servicios de consultoría de hardware y software o el procesamiento de datos, por poner algunos ejemplos.

\section{Colaboración internacional, conocimiento y resultados innovadores en las KIBS}

Las KIBS necesitan renovar sus estructuras de conocimiento para seguir siendo innovadoras $\mathrm{y}$ se ha comprobado que las mejoras e introducciones de nuevos servicios están asociadas al acceso a fuentes externas de conocimiento (Leiponen, 2005). Además de ser empresas muy innovadoras, las KIBS son transmisoras y facilitadoras de conocimiento e incluso fuentes de innovación (Hertog, 2000). Por ello, es importante entender los factores clave que determinan la mejora de sus procesos y la generación de nuevos servicios; en concreto, el papel de la colaboración tecnológica internacional atendiendo a dos dimensiones: (i) la proximidad entre los socios y (ii) la diversidad de conocimiento entre ellos.

El concepto de proximidad lleva inherente muy distintas dimensiones, si bien, tendemos a asociarlo a la distancia geográfica. Sin embargo, la proximidad va más allá de esta dimensión para incluir otros factores tan importantes como la dimensión cognitiva, organizativa, social e institucional, que a menudo se capturan implícitamente con la proximidad geográfica. Así, se ha demostrado que la proximidad en los clusters industriales favorece la interacción entre empresas, permite compartir experiencias, información o infraestructuras $\mathrm{y}$, con todo, mejorar la innovación. Son muy diversos los ejemplos en este sentido, siendo un caso bien representativo el de Silicon Valley en California.

En cuanto a la diversidad de conocimiento, se ha probado como la diversidad de los socios es un factor positivo en la colaboración tecnológica, al permitir el acceso a un conocimiento heterogéneo y/o complementario y expandir la base de conocimiento de las empresas (Nieto \& Santamaría, 2007). Por otra parte, la diversidad tecnológica entre las empresas conlleva dificultades en compartir conocimiento y el aprendizaje de los socios (Oxley \& Sampson, 2004). Estos problemas se amplifican en un contexto internacional, porque la posesión de conocimiento muy distinto puede dificultar su asimilación por los socios.

\section{Características del conocimiento y colaboración internacional en P-KIBS y T-KIBS}

Por sus características, el conocimiento podrá ser más o menos tácito, afectando con ello al grado de codificabilidad (Vissers \& Dankbaar, 2013). Ese grado de codificabilidad del conocimiento influirá en su potencial para poder ser transferido cuando los socios estén separados geográficamente (Howells, 2002). Así, cuando queremos analizar el efecto de la colaboración internacional en distintos sectores como P-KIBS y T-KIBS, es crucial ser consciente de la heterogeneidad de sus sistemas de conocimiento - particularmente en lo relativo al grado de codificabilidad del conocimiento y a la especificidad del contexto. 
En las P-KIBS, una elevada proporción del conocimiento reside en los individuos, quienes desarrollan técnicas de resolución de problemas para clientes específicos. Por ejemplo, Accenture y el National Bank de Canadá $^{2}$ combinan sus habilidades para implementar una solución innovadora para convertirse en el banco más rápido para los pequeños negocios en Canadá. Gran parte del conocimiento depende de las personas y se puede generar mientras se presta el servicio. Por tanto, se trata de un conocimiento con raíces en contextos muy específicos. Es por tanto un conocimiento de naturaleza más tácita, difícil de codificar, ampliamente enraizado en habilidades y prácticas de individuos.

En T-KIBS se trata con una mayor proporción de conocimiento técnico y experimental. Este tipo de conocimiento es relativamente más fácil de transferir porque suele ser interpretado de forma similar entre distintos contextos (incluso en distintos países) (Robertson y otros, 2003). Al ser menos dependientes del contexto, las tareas son más fácilmente sistematizables y el grado de discrecionalidad profesional es menor que en PKIBS (Consoli \& Elche-Hortelano, 2010). Son numerosas las colaboraciones en el ámbito de la biomédica y la salud que han visto la luz con la reciente pandemia de la COVID-19. Este es el caso, por ejemplo, de Achilles Therapeutics colaborando con otra empresa biotecnológica, Ori Biotech para innovar y mejorar la efectividad de la terapia de células. ${ }^{3}$

Las diferencias en la naturaleza del conocimiento, la forma de organizarlo y crearlo, harán que las vías para conseguir innovar en cada sector también sean diferentes (Pina \& Tether, 2016). Por eso, es esperable que la colaboración internacional tenga distintas consecuencias en la innovación, y sean diferentes en función de la proximidad entre los socios y la diversidad del conocimiento.

\section{P-KIBS y la importancia de proximidad entre los socios}

El estudio revela que la proximidad entre los socios de la colaboración internacional es un factor que incrementa la probabilidad de innovar para las P-KIBS. Si pensamos a qué puede obedecer este hecho, debemos considerar las ventajas competitivas que pueden tener las $\mathrm{P}$ KIBS y la naturaleza del conocimiento que manejan para desempeñar su actividad.

Las actividades que realizan son altamente dependientes del contexto, lo que genera información muy específica del problema y/o requerimientos de cada cliente. Eso hace que sean actividades más difíciles de estandarizar y replicar. Si contamos con un conocimiento más tácito e individual será difícil trasladarlo desde los individuos a la organización y, más aún, compartirlo con otras empresas, sobre todo cuando hay una separación geográfica. El conocimiento altamente incorporado en las personas requiere de un mayor nivel de interacción entre los individuos que lo poseen y una colaboración que precisa de contacto directo y cercanía. La distancia geográfica entre los socios limita esta interacción, por lo que se hace más difícil compartirlo.

Por otro lado, las actividades profesionales son altamente dependientes del contexto social e institucional en el que opera la empresa (Miles, 2005). Si estas actividades están relacionadas con problemas específicos del cliente, la interacción social y la experiencia práctica son particularmente útiles en el contexto en que se desarrollan. Sería el caso de las empresas de consultoría legal o agencias de investigación de mercados. Por contra, colaborar

\footnotetext{
2 https:/www.newswire.ca/news-releases/accenture-and-ncino-help-national-bank-transform-its-commercial-lending-platform898118105.html.

${ }^{3} \mathrm{https}$ //www.pinsentmasons.com/out-law/analysis/pandemic-collaborations-life-sciences.
} 
internacionalmente con socios de muy distintos contextos institucionales reduce el valor del conocimiento que puede llegar a compartirse entre los socios, y con ello la capacidad de innovar.

Así, la proximidad en las actividades de colaboración de las P-KIBS aporta claras ventajas. Coherente con ello, los resultados que se obtienen del estudio reflejan que, para las PKIBS, las innovaciones de servicio y de proceso se ven favorecidas por la colaboración entre empresas nacionales. En cambio, en el plano internacional sólo se ve un impacto positivo para el caso de los socios europeos. La diversidad entre los socios no contribuye favorablemente a las innovaciones en las P-KIBS.

\section{T-KIBS y la importancia de la diversidad de conocimiento}

El estudio ilustra la importancia de la diversidad del conocimiento para el éxito de la colaboración internacional en el contexto de las T-KIBS. Para entender este resultado, debemos partir del hecho de que las T-KIBS ofrecen servicios relacionados con la tecnología, por lo que su ventaja competitiva está relacionada con la disponibilidad de conocimiento y la explotación de avances tecnológicos (Miles, 2005). Unido a ello, el conocimiento empleado en este tipo de servicios es más explícito, codificable y modular. En este sentido es más fácil de transferir dentro de la organización y a otros socios en la colaboración. Desde un punto de vista social, el conocimiento no depende tanto de la interacción con los clientes y del contexto, es más neutral y puede ofrecerse en servicios más estandarizados (Consoli \& Elche-Hortelano, 2010). Acceder a una diversidad de socios internacionales que faciliten tecnologías más avanzadas, permitirá a las T-KIBS beneficiarse de un conocimiento altamente codificado y aplicable a su realidad concreta. Un ejemplo de empresas de este tipo de servicios lo encontramos en la americana nCino que ofrece tecnología a nivel global con su software y servicios tecnológicos para los sistemas operativos de bancos. 4

Los resultados del estudio confirman el beneficio de esta diversidad en la innovación de las T-KIBS, tanto en las innovaciones de servicio como de proceso. Las empresas que colaboran con socios de distintas regiones a nivel internacional alcanzan mejores resultados de innovación en servicio y en proceso, mostrando que son capaces de sacar partido a la heterogeneidad del conocimiento. Además, se obtiene evidencia sobre cómo la colaboración con socios de otras regiones más allá de Europa y EEUU, en principio más alejados geográfica e institucionalmente, tiene efectos positivos para la innovación de servicios de las T-KIBS.

\section{Conclusiones}

El estudio confirma el impacto de la colaboración internacional en las KIBS y cómo difiere dependiendo de la actividad concreta que desempeña la empresa. La diversidad de conocimiento es útil para generar nuevas ideas e innovaciones, pero sacarle partido estará condicionado por la facilidad de compartir dicho conocimiento y la dependencia del contexto asociada a cada actividad.

En este sentido, las P-KIBS se benefician más de las colaboraciones nacionales $\mathrm{y}$, en el caso de las internacionales, aquéllas que se llevan a cabo con socios más próximos (situados en el contexto de la Unión Europea). Sin embargo, las T-KIBS aprovechan la heterogeneidad de los socios con más facilidad $\mathrm{y}$, por ello, se benefician de colaboraciones internacionales más diversas y no necesariamente tan próximas.

\footnotetext{
${ }^{4}$ Véase www.ncino.com
} 


\section{Implicaciones para la gestión}

En el diseño de una colaboración tecnológica, es crucial la selección del socio. Para la empresa, ello conlleva el manejo de mucha información: interna, de los potenciales socios y del contexto en el que se va a desarrollar la colaboración. En este trabajo se apunta a varios factores que pueden ayudar a entender el éxito de la colaboración internacional, atendiendo a la actividad de la empresa y al origen internacional de los socios. La proximidad en los socios internacionales $\mathrm{y}$, con ello, su cercanía geográfica e institucional son factores importantes para las empresas de servicios profesionales. Por su parte, las empresas de servicios relacionados con la tecnología encuentran una mayor contribución a la innovación cuando buscan socios de diferentes ubicaciones geográficas. Estos aspectos cobran especial importancia tras la pandemia mundial que ha provocado la COVID-19, ya que muchas de las colaboraciones se han tenido que materializar a distancia y mediante métodos virtuales.

\section{Referencias}

Alvesson, M. (2004). Knowledge work and knowledge intensive firms. Oxford: Oxford University Press.

Consoli, D., \& Elche-Hortelano, D. (2010). Variety in the knowledge base of Knowledge Intensive Business Services. Research Policy, 39(10), 1303-1310.

Eurostat, (2017). Structural business statistics overview of the business economy. Retrieved from https://ec.europa.eu/eurostat/statistics-
explained/index.php?title=Structural_business_statist ics_overview\#Sectoral_analysis

Hertog, P.D. (2000). Knowledge-intensive business services as co-producers of innovation. International Journal of Innovation Management, 4(4), 491-528.

Howells, J. (2002). Tacit Knowledge, Innovation and Economic Geography. Urban Studies, 39(5-6), 871884.

Leiponen, A. (2005). Organization of knowledge and innovation: the case of Finnish business services. Industry and Innovation, 12(2), 185-203.

Miles, I. (2005). Knowledge intensive business services: prospects and policies. Foresight, 7(6), 3963

Nieto, M.J., \& Santamaría, L. (2007). The importance of diverse of collaborative networks for the novelty of product innovation. Technovation, 27, 367-377.

Oxley, J. E., \& Sampson, R.C. (2004). The scope and governance of international R\&D alliances. Strategic Management Journal, 25, 723-749.

Pina, K., \& Tether, B.S. (2016). Towards understanding variety in knowledge intensive business services by distinguishing their knowledge bases. Research Policy, 45(2), 401-413.

Robertson, M., Scarbrough, H. \& Swan, J., (2003). Knowledge creation in professional service firms: Institutional effects. Organization Studies, 24(6), 831857.

Rodríguez, A., Nieto, M.J., \& Santamaría, L. (2018). International collaboration and innovation in professional and technological knowledge-intensive services. Industry and Innovation, 25(4), 408-431.

Vissers, G., \& Dankbaar, B. (2013). Knowledge and proximity. European Planning Studies, 21(5), 700721. 